\title{
THREE-DIMENSIONAL ACOUSTIC TISSUE MODEL: A COMPUTATIONAL TISSUE PHANTOM FOR IMAGE ANALYSES
}

\author{
J. Mamou, M.L. Oelze, W.D. O’Brien, Jr., J.F. Zachary \\ Department of Electrical and Computer Engineering, University of Illinois at Urbana- \\ Champaign; Department of Bioengineering, University of Illinois at Urbana-Champaign; \\ Department of Veterinary Pathobiology, University of Illinois at Urbana-Champaign
}

\begin{abstract}
A novel methodology to obtain three-dimensional (3D) acoustic tissue models (3DATMs) is introduced. 3DATMs can be used as computational tools for ultrasonic imaging algorithm development and analysis. In particular, 3D models of biological structures can provide great benefit to better understand fundamentally how ultrasonic waves interact with biological materials. As an example, such models were used to generate ultrasonic images that characterize tumor tissue microstructures. 3DATMs can be used to evaluate a variety of tissue types. Typically, excised tissue is fixed, embedded, serially sectioned, and stained. The stained sections are digitally imaged (24-bit bitmap) with light microscopy. Contrast of each stained section is equalized and an automated registration algorithm aligns consecutive sections. The normalized mutual information is used as a similarity measure, and simplex optimization is conducted to find the best alignment. Both rigid and non-rigid registrations are performed. During tissue preparation, some sections are generally lost; thus, interpolation prior to $3 \mathrm{D}$ reconstruction is performed. Interpolation is conducted after registration using cubic Hermite polynoms. The registered (with interpolated) sections yield a $3 \mathrm{D}$ histologic volume (3DHV). Acoustic properties are then assigned to each tissue constituent of the 3DHV to obtain the 3DATMs. As an example, a 3D acoustic impedance tissue model (3DZM) was obtained for a solid breast tumor (EHS mouse sarcoma) and used to estimate ultrasonic scatterer size. The 3DZM results yielded an effective scatterer size of $32.9 \quad( \pm 6.1) \mu \mathrm{m}$. Ultrasonic backscatter measurements conducted on the same tumor tissue in vivo yielded an effective scatterer size of $33( \pm 8) \mu \mathrm{m}$. This good agreement shows that 3DATMs may be a powerful modeling tool for acoustic imaging applications
\end{abstract}

Key words: Registration; 3D reconstruction; tissue characterization 


\section{INTRODUCTION}

This contribution presents a set of strategies to reconstruct three-dimensional (3D) acoustic tissue models (ATMs) from consecutive two-dimensional (2D) stained histologic sections of a mouse sarcoma tumor. The 3D reconstruction strategies are familiar to the medical imaging and image/signal processing communities. Tissue preparation (sectioning, etc.) is challenging and leads to a 3D dataset with artifacts that need to be accounted for in order to obtain accurate 3D models. Specifically, all the sections do not have the same contrast. Also, during imaging the pathologist manually aligns one section to the next consecutive. This alignment is approximate and fine-tuning registration needs to be conducted. Finally, during sectioning of the tissue some of the sections are lost. Therefore, sections need to be interpolated post registration for accurate reconstruction of $3 \mathrm{D}$ volumes. Thus, this study addresses signal and image processing strategies to obtain accurate 3D histologic volumes (3DHV) from the 2D histologic sections.

The reconstructed 3DHVs can be used as 3D computational phantoms for different wave propagation simulations and as imaging algorithm development tools. For example, 3DHVs are converted to 3DATMs by assigning acoustic properties to every voxel (i.e., speed of sound, density, attenuation, etc.), then computational algorithms could be used to solve acoustic wave equations to study wave propagation phenomena in tissue. Furthermore, techniques were recently developed to estimate ultrasonic scatterer properties from 3D impedance maps (i.e., 3DATMs where every voxel was assigned an acoustic impedance value) [1]. These estimates are believed to have diagnostic potential for detecting and diagnosing disease. Scatterer size estimates were obtained from the 3D impedance map (3DZM) obtained from the mouse sarcoma and compared to ultrasonic measurements obtained in situ from the same tumor.

This 3D reconstruction study was conducted as a first step of a very ambitious project that is trying to establish connections between ultrasonic scattering signatures and tissue microstructure for noninvasive diagnostics. Hence, 3DATMs can be used for better understanding the complicated interactions of ultrasound waves with biological material.

\section{TISSUE PREPARATION AND IMAGING}

Three-dimensional reconstruction strategies are developed and demonstrated with a murine sarcoma tumor model. The EHS (Englebreth-Holm-Swarm) cell line (ATCC - CRL-2108, American Type Culture Collection) is a transplantable sarcoma in C57BL/6 mice. This tumor grows subcutaneously 
and produces extracellular matrix (ECM) components such as laminin, collagen IV, entactin, and heparan sulfate proteoglycan. The tumor was allowed to grow until it was of sufficient size for harvesting.

Sections of the EHS sarcoma were fixed by immersion in $10 \%$ neutralbuffered formalin, embedded in paraffin, sectioned at $3-\mu \mathrm{m}$ thickness, mounted on glass slides, and stained with hematoxylin and eosin (H\&E) for evaluation. The processes of fixation, paraffin infiltration, and sectioning of the EHS sarcoma for histopathologic evaluation causes a minor degree of inherent tissue shrinkage that is small, but indeterminate for each section. In addition, it is not possible to obtain 200 serial sections (one section after another) without the loss of some sections in the series due to technical difficulties in tissue sectioning. EHS sarcoma sections, mounted on glass slides, were examined and captured using a Nikon Optophot-2 light microscope equipped with a Sony color video camera.

A board-certified pathologist via a microscope stage manually registered each image with the proceeding image. This manual registration process is very labor-intensive and imprecise; therefore, an automatic registration algorithm was developed. For the EHS dataset, the optical images measured laterally $400 \mu \mathrm{m}$ by $300 \mu \mathrm{m}$. The EHS dataset contained 200 sections of which 54 sections $(26 \%)$ were lost during tissue preparation. Each bitmap image measured 640 pixels by 480 pixels with 8-bit accuracy for each red, green and blue (RGB) component.

\section{THREE-DIMENSIONAL RECONSTRUCTION}

Obtaining a 3DATM (or a 3DZM) from 2D sections involves four signal and image processing steps. 1) The contrast of the sections is equalized because the uptake of stain, in general, is not uniform; nor is the video capture intensity necessarily the same from section to section. 2) A fine-tuning registration is conducted. 3) For the missing sections, interpolation is performed. 4) The resulting $3 \mathrm{DHV}$ is converted to a 3DZM by assigning an acoustic impedance value to each voxel.

\subsection{Contrast Equalization}

Consecutive histologic sections generally have different contrasts. Each section may have been stained for a slightly different duration and/or may be slightly different in thicknesses, thus resulting in variability of stain uptake. Equalization was conducted for each of the three-color components (i.e., red, green and blue) of each image. 
Specifically, let $H_{c}$ denote the cumulative histogram of image I (for one of the color components). Thus, $H_{c}(\alpha)$ is the number of pixels with intensity level $\leq \alpha$ in the image. Then, the equalizing transform is given by:

$$
\alpha^{\prime}=255 \frac{H_{C}(\alpha)}{N} \text {, }
$$

where $N$ is the total number of pixels in the image and the 255 factor has been added so that the new intensity is in the range 0-255 (because $N \geq H_{c} \geq 0$ ). $\alpha^{\prime}$ is the new intensity value for of the pixels that had an intensity value of $\alpha$.

\subsection{Registration}

Registration or alignment is an essential step in the 3D reconstruction because if the consecutive sections are not aligned correctly, then the resulting 3DATM will yield misleading or incorrect results. For our 3D modeling, each section needs to be aligned with the next. Specifically, we are trying to determine the best transformation to apply to an image $I_{2}$ so that it is the most similar to the original and adjacent image $I_{1}$. Hence, the registration algorithm is composed of three main parts: set of transformations, similarity measure and optimization.

The rigid transformation set, $S_{r}$, is composed of rotations and translations (it has three degrees of freedom (DOFs), two translational and one rotational). The non-rigid transformation set, $S_{n r}$, is composed of just about any conceivable adjustable parameter. In the present work $S_{r}$ was used as it is a reasonable choice considering the error sources in the initial manual alignment of the images. However, a subset of $S_{n r}$ limited to the affine transformations $\left(S_{a f}\right)$ was also considered because this class of transforms (6 DOFs) can in particular compensate for the unknown inherent tissue shrinkage and shearing that occur during tissue preparation.

Once the image $I_{2}$ is transformed it is necessary to quantify how $T\left(I_{2}\right)$ is similar to $I_{1}$. Let us define $F(I, J)$ as a function that quantifies the similarity between image $I$ and image $J$. Many choices are possible for $F$. However, it has been shown that the functions $F$ based on information theory concepts (entropy) are usually very robust. Thus, the normalized mutual information (NMI) was used as a similarity measure [2].

The next step is to update the transform $\left(T \rightarrow T^{\prime}\right)$ so that $F\left(I_{1}, T\left(I_{2}\right)\right)>F\left(I_{1}, T^{\prime}\left(I_{2}\right)\right)$, where $T$ and $T^{\prime}$ belong to the set of transforms. Many optimization algorithms rely on the possibility of evaluating or easily estimating the gradient and/or the Hessian of the similarity function (with 
respect to the transformation's DOFs). These techniques are not readily applicable to the NMI measure. Popular algorithms that bypass the need for derivatives are Powell's algorithm and the simplex method. Powell's algorithm optimizes each of the transformation's DOFs one by one. The simplex method considers all DOFs simultaneously. The latter was used in the present work [3].

\subsection{Missing Section Interpolation}

When tissue is sectioned, some sections are lost or destroyed. Therefore, it is necessary to interpolate the missing sections to avoid further artifacts in the 3D tissue model. To interpolate the missing voxels, cubic Hermite polynomials were used. Specifically, the 3D interpolation was first reduced to several 1D interpolations by considering the 1D signal formed by following the intensity at the same pixel location from one section to the next (and for each of the three color bands). Thus, this 1D signal was regularly sampled but has missing samples. To interpolate missing samples, the Hermite interpolation searches for a third-order polynomial (i.e., four unknowns) to match the value and the slopes of the signal on both sides of the "hole". Thus, the scheme leads to four equations to find four unknowns.

\subsection{Acoustic Impedance Assignment}

To convert each pixel value to appropriate acoustic impedance value a colorthreshold algorithm was used on every H\&E stained bitmap image. Tissue proteins stained with eosin have shades of color ranging from very light pink to dark pink, depending on the concentration of protein within the structure. This differential staining allowed for the recognition of unique microstructures in the tissue. A range of colors corresponding to an identified microstructure in the stained bitmap images was then assigned a single color. The new single color was thus representative of the acoustic impedance for that tissue microstructure. For each pixel of the same color in the color-thresholded bitmap image the same acoustic impedance value was assigned.

\section{SCATTERER SIZE ESTIMATION}

The backscattered intensity, $I_{b s}$, due to an incident plane wave on a weakly inhomogeneous medium can be written as [4]: 


$$
I_{b s}=A k^{4}\left|\iiint \frac{\Delta z}{z} e^{-2 i k r_{0}} d v_{0}\right|^{2}
$$

where $\mathrm{A}$ is proportionality constant and $\mathrm{z}$ is the acoustic impedance. The power spectrum of the relative impedance variations can be easily extracted from the backscattered intensity. A Gaussian form factor was chosen to model ultrasonic scattering because it is the form factor chosen for ultrasonic evaluation of the sarcoma. The scatterer size is then estimated by fitting the Gaussian FF to the power spectrum of the 3DZM [1].

\section{RESULTS}

The 3D reconstruction strategies were applied to the EHS dataset. Figure 1a shows 43 consecutive sections of the original EHS dataset. These sections were subimages of size $218 \mu \mathrm{m}$ by $156 \mu \mathrm{m}$ (i.e., 350 by 250 pixels) of the original $400 \mu \mathrm{m}$ by $300 \mu \mathrm{m}$ EHS images. Among these 43 consecutive sections, 7 were missing (including 4 consecutive sections). The sections were misaligned and the contrast between sections was also slightly different. The misalignment can be observed by following the pink quasicircular structure (muscle) from one section to the next. Also, sections 8 and 17 have slightly better contrast than their neighboring sections.

Figure $1 \mathrm{~b}$ shows the reconstructed 43 -section dataset. To align the images, affine registration was used. Figure $1 \mathrm{~b}$ shows that all the reconstructed images have a very similar contrast, that is, none of the sections visually stand out as clearly darker or lighter than the others. The sections were also aligned, as can be seen by following the oblique structures in the top left corner. The interpolated missing sections were of lower quality than the others; note that the interpolated missing sections were not as sharp and contained some edge artifacts, particularly for the four reconstructed images of the four consecutive missing sections.

Figure 2a shows a 3D rendering of the 3DZM from the 43-section dataset of Figure 1b. Figure 2b shows the deduced 3DZM for which seven distinct impedance values were used: 2.00 Mrayl for the nuclear heterochromatin (black), 1.85 Mrayl for the nuclear euchromatin (blue), 1.70 and 1.65 Mrayl for the extracellular matrix (dark red/red), 1.60 for the vascular space/whole blood (white), and 1.58 and 1.55 for the cytoplasm (green/yellow).

The scatterer size estimation was then conducted on the 3DZM (Figure 2b). The 3DZM results yielded an effective scatterer size of $32.9( \pm 6.1) \mu \mathrm{m}$. 
Ultrasonic backscatter measurements conducted on the same tumor tissue in vivo yielded an effective scatterer size of $33( \pm 8) \mu \mathrm{m}$.

a)

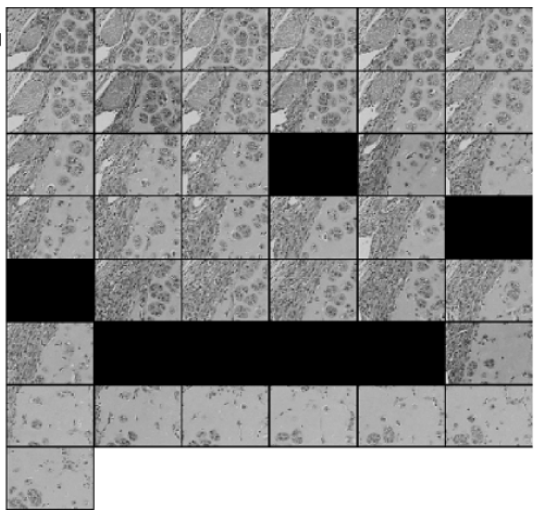

b)

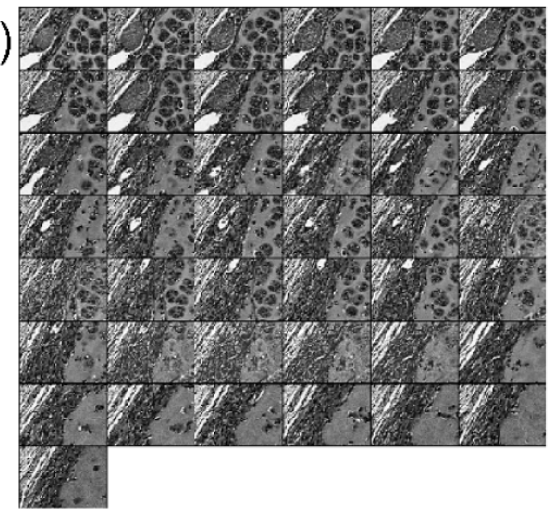

Figure 1. a) Forty-three-section dataset from the EHS dataset. Sections are of size $218 \mu \mathrm{m}$ by $156 \mu \mathrm{m}$ (i.e., 350 by 250 pixels). Sections are subimages of size $218 \mu \mathrm{m}$ by $156 \mu \mathrm{m}$ (i.e., 350 by 250 pixels) of the original $400 \mu \mathrm{m}$ by $300 \mu \mathrm{m}$ EHS sections. The sections were consecutive from left to right and top to bottom. Black fields symbolize missing sections.

b) Reconstruction of the 43-section EHS dataset. Contrast of the available sections was equalized. Each section was affine-registered with the next available section. Missing sections were Hermite-interpolated post equalization and post registration.
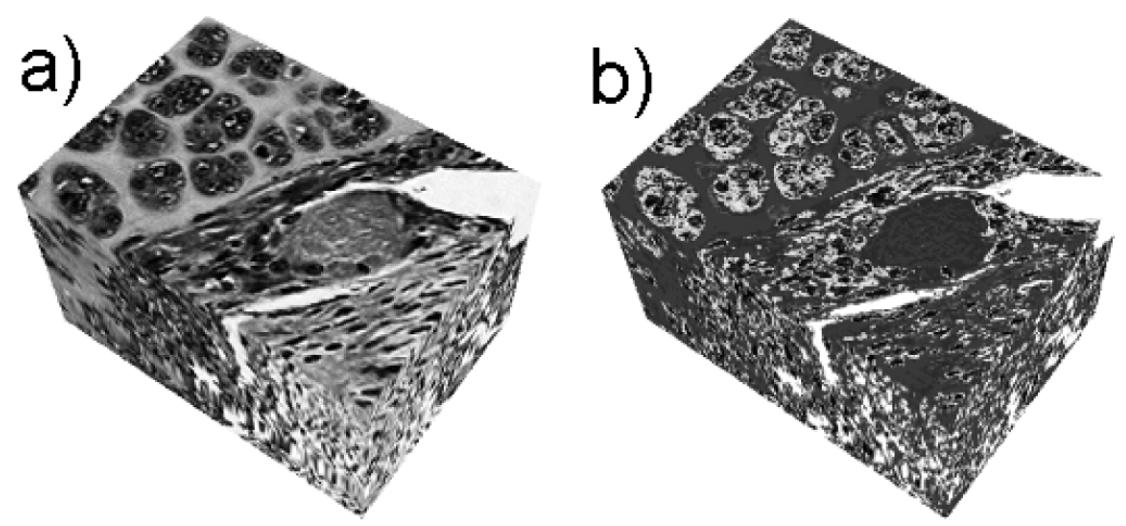

Figure 2. a) 3D rendering from the reconstructed 43-section dataset (Fig. 1b). Volume is of size $218 \mu \mathrm{m}$ by $156 \mu \mathrm{m}$ by $129 \mu \mathrm{m}$ (depth). b) 3D rendering of the deduced 3DZM. The 3DZM was deduced by using a 7-level color-threshold algorithm on each reconstructed section. 


\section{CONCLUSIONS}

A new set of strategies was proposed to obtain 3D acoustic tissue models from adjacent histologic sections. The strategies were illustrated using a 200 -section dataset from a mouse sarcoma tumor. Imperfections of the original histologic dataset were carefully accounted for. First, contrast of all the sections was equalized and affine registration was conducted to ensure that all the sections were perfectly aligned. Then, sections lost during tissue preparation were interpolated. Finally, to deduce a 3DZM from this $3 \mathrm{DHV}$ acoustic impedance values were assigned using a 7-level threshold algorithm. An ultrasonic tissue characterization scheme was developed to obtain ultrasonic scatterer size estimates from the 3DZM. 3DZM estimates for the sarcoma 3DZM were found to be virtually the same as those obtained on the same sarcoma with ultrasound. This excellent agreement demonstrates that 3DATMs may be a powerful modeling tool for acoustic imaging applications.

\section{ACKNOWLEDGEMENT}

The authors would like to thank James P. Blue. Work supported by the University of Illinois Research Board.

\section{REFERENCES}

1. J. Mamou, M.L. Oelze, W.D. O’Brien, Jr., and J.F. Zachary, "Identifying ultrasonic scattering sites from 3d impedance maps," J. Acoust. Soc. Am., pp. 413-423, 2005.

2. C. Studholme, D.L.G. Hill., and D.J. Hawkes, "An overlap invariant entropy measure of 3d medical image alignement," Pattern Recogn., vol. 32, pp. 71-86, 1999.

3. J.A. Nelder., and R. Mead, "A simplex method for function minimization," Computer J., vol. 7, pp. 308-313, 1965.

4. P.M. Morse., and K.U. Ingard, Theoretical acoustics, McGraw-Hill, New York, NY, 1968. 